\title{
Modelo RE-AIM: Tradução e Adaptação cultural para o Brasil
}

\section{RE-AIM framework: Translation and Cultural adaptation to Brazil Modelo RE-AIM: Traducción y Adaptación Cultural para el Brasil}

\author{
Recebido: 16/08/2013 \\ Aprovado: 05/11/2013
}

\author{
Fabio Araujo Almeida ${ }^{1}$ \\ Fabiana Almeida Brito ${ }^{2}$ \\ Paul Andrew Estabrooks ${ }^{3}$
}

O propósito deste artigo é apresentar a tradução e a adaptação do modelo RE-AIM (Reach = Alcance, Eficácia/Efetividade, Adoção, Implementação, Manutenção) para a realidade brasileira por meio do processo de tradução, retrotradução, adaptação cultural e posterior avaliação de equivalência por especialistas, amostra da população-alvo e equipe de pesquisadores. O RE-AIM visa instrumentalizar gestores de programas, avaliadores, leitores de artigos científicos e fontes financiadoras com ferramentas e estratégias, destacando elementos essenciais de programas para adoção sustentável e implementação eficaz focados na prevenção de agravos e promoção da saúde, podendo ser aplicado em todos os âmbitos do Modelo Socioecológico, atentando para todos os níveis de influência que podem ser abordados a fim de se apoiar as escolhas de estilo de vida saudável em longo prazo. O RE-AIM proporciona um método para avaliar o potencial ou o real impacto na saúde coletiva em programas e intervenções com capacidade para colaborar na consolidação do SUS na prática.

Descritores: Avaliação em Saúde; Programas, Tradução; Adaptação; Comparação Transcultural.

The purpose of this article is to present the translation and adaptation of the RE-AIM framework (Reach, Efficacy/Effectiveness, Adoption, Implementation, Maintenance) for Brazilian reality. Through a process of translation, back translation, cultural adaptation and subsequent equivalence assessment by experts, sample of the target population and the research team. The RE-AIM aims to equip program managers, evaluators, readers of scientific articles, and funding sources with tools and strategies. Highlighting key elements of sustainable programs for adoption and effective implementation focused on prevention and health promotion. RE-AIM can be applied at all levels of the socio-ecological model attempting to influence healthy lifestyle choices in the long term. The RE-AIM provides a method to assess the potential or actual impact on public health programs and interventions with great potential to assist in the consolidation of system public of health Brazilian in practice.

Descriptors: Health Evaluation; Programs, Translating; Adaptation; Cross-Cultural Comparison.

El propósito de este artículo es presentar la traducción y adaptación del modelo RE-AIM (Reach=Alcance, Eficacia/efectividad, Adopción, Implementación, Mantenimiento) para la realidad brasileña. Mediante un proceso de traducción, traducción inversa, adaptación cultural y la posterior evaluación de la equivalencia de los expertos, muestra de la población objetivo y el equipo de investigación. El RE-AIM pretende dotar a los directores de programas, evaluadores, y lectores de artículos científicos con estrategias apuntando los elementos clave para la adopción y aplicación eficaz de programas centrados en la prevención de enfermidades y la promoción de la salud. Se puede aplicar en todos los niveles del modelo socio-ecológico con el fin de apoyar las elecciones de estilo de vida saludable en el largo plazo. El RE-AIM proporciona un método para evaluar el impacto potencial o real de los programas de salud pública y las intervenciones con potencial para contribuir a la consolidación del sistema publico de salud brasileño en la práctica.

Descriptores: Evaluación en Salud; Programas, Traducción; Adaptación; Comparación Transcultural.

\footnotetext{
1 Assistente Social. Especialista em Práticas Comunitárias. Mestre em Serviço Social com ênfase em Prática Comunitária e Desenvolvimento Internacional. Doutor em Serviço Social. Professor Adjunto do Departamento de Nutrição Humana, Alimentos e Exercício da Universidade Virginia Tech, Virgínia, Estados Unidos da América. falmeida@vt.edu

2 Enfermeira. Especialista em Atenção Primária e Saúde da Família. Mestre em Atenção à Saúde. Doutoranda do Programa de Educação de Pós-Graduação Interdisciplinar (IGEP) em Pesquisa Translacional de Obesidade no Departamento de Nutrição Humana, Alimentos e Exercício na Universidade Virginia Tech, Virgínia, Estados Unidos da América. fabiana@vt.edu ${ }^{3}$ Educador Físico. Mestre em Ciência com ênfase em Cinesiologia. Doutor em Cinesiologia. Professor Titular do Departamento de Nutrição Humana, Alimentos e Exercício na Universidade Virginia Tech. Virgínia, Estados Unidos da América. Membro da equipe desenvolvedora do RE-AIM.
} 


\section{INTRODUÇÃO}

0 Sistema Único de Saúde (SUS) no Brasil se alicerça na saúde como um direito humano, com determinantes sociais e políticos amplos, necessitando de políticas de desenvolvimento amparadas por regulamentos e recursos inclusivos, dinâmicos e transparentes ${ }^{1}$. Assim, a prevenção de agravos e a promoção da saúde são o principal foco da Atenção Primária à Saúde (APS). A APS, no modelo de saúde brasileiro, possui valores, princípios e elementos próprios como forma de organização dos serviços de saúde e constitui o primeiro contato com o sistema de saúde, sendo responsável pela organização do cuidado à saúde individual, familiar e coletiva, norteando a distribuição de recursos a fim de melhorá-la².

A avaliação e o monitoramento devem servir como ferramenta para a qualidade de gestão e planejamento das ações em saúde para garantir uma melhor distribuição de recursos. A avaliação provém da aferição de resultados das suas ações em saúde para identificar os pontos de intervenção, por meio de estudos comparativos com outras situações ou parâmetros, levando ao questionamento, à revisão das normas estabelecidas e aos processos de produção em saúde ${ }^{3}$. Para a garantia da atenção à saúde, faz-se necessário um planejamento com base no estudo de necessidades em saúde da população $0^{4}$, pois o SUS deve ser utilizado de maneira racional para que consiga executar seus princípios e diretrizes.

Como tal, programas e políticas no âmbito do SUS que visam ter impacto na saúde da população devem se concentrar em estratégias baseadas em evidências com base em uma variedade de perspectivas, incluindo comportamentos individuais de saúde, culturais e sociais 5 .

No decorrer dos últimos anos, uma série de políticas e programas ${ }^{4,6,7}$ foi criada no Brasil em uma tentativa de responder à atual situação epidemiológica marcada por condições que necessitam de manejo contínuo. Isso porque as doenças crônicas não transmissíveis são responsáveis por $72 \%$ das causas de óbitos, estando entre as principais causas de internações hospitalares e representando um impacto crescente nos custos diretos ${ }^{8}$.

No entanto, mesmo com esse aumento crescente de programas e iniciativas $^{4,6,7}$, a prevalência de doenças crônicas não transmissíveis no Brasil continua a crescer em taxas alarmantes, e agora está impactando aqueles que são de baixo nível socioeconômico, mais vulneráveis e minorias ${ }^{8}$. Há uma necessidade urgente de se traduzir, adaptar e difundir programas baseados em evidências na prática.

Há poucas dúvidas de que o comportamento humano é fundamental para a saúde populacional ${ }^{9}$. Por exemplo, aqueles que são fisicamente ativos, comem saudavelmente e mantêm um peso corporal adequado são menos suscetíveis a doenças, e os doentes que se dedicam a esses comportamentos têm evolução de suas doenças menos graves, quando comparados a pessoas que não têm esses fatores ${ }^{10}$. Como resultado, ao longo das últimas quatro décadas houve ampliação de pesquisas aferindo a eficácia de diferentes intervenções de mudanças comportamentais ${ }^{11}$.

Ainda assim, há pouca evidência de pesquisas baseadas em intervenções sendo traduzidas para a prática clínica ou comunitária. Para abordar esse problema, Glasgow e sua equipe ${ }^{12}$ sugeriram que o sucesso das intervenções de mudanças comportamentais deve ser avaliado com base tanto em fatores individuais quanto organizacionais. 0 modelo RE-AIM $($ Reach $=$ Alcance, Eficácia/Efetividade, Adoção, Implementação, Manutenção) foi introduzido como um método para equilibrar o foco de pesquisas tanto sobre a validade interna quanto externa, incluindo resultados que são operacionalizados em níveis individuais, organizacionais ou ambos.

Desde a sua criação original, o modelo RE-AIM continua a evoluir e tem sido aplicado numa variedade de esferas com 


\section{Administração em Saúde Coletiva}

inúmeras populações diferentes. 0 modelo RE-AIM tem sido usado não só para organizar revisões da literatura ${ }^{12}$, para ajudar a planejar e melhorar as chances de implementação de programas em organizações ${ }^{13,14}$, para comparar os pontos fracos e fortes de programas concorrentes ${ }^{15}$, mas também como uma ferramenta para o planejamento de programas e avaliação ${ }^{16}$.

Atualmente, o modelo RE-AIM tem como foco proporcionar aos gestores de programas, avaliadores, leitores de artigos científicos e fontes financiadoras ferramentas e estratégias para ajudar a destacar elementos essenciais de programas. Elementos esses que podem ajudar na adoção sustentável e na implementação de programas eficazes focados na prevenção de agravos e na promoção da saúde ${ }^{17}$.

No entanto, a aplicação integral do modelo RE-AIM à realidade brasileira requer mais do que a simples tradução linguística (inglês para português). Exige um processo de tradução, retrotradução, adaptação cultural e posterior avaliação antes da aplicação completa do modelo. Vários autores têm destacado a importância da tradução e da adaptação cultural de instrumentos e modelos ${ }^{18-20}$, uma vez que a elaboração de novos instrumentos e modelos requer muito tempo, recursos e esforços ${ }^{18}$. Ressalta-se que a utilização de modelos elaborados em outros contextos culturais deve reconhecer as diferenças entre definições, crenças e comportamentos relacionados a um construto e impõe que seja precedida de uma avaliação meticulosa da equivalência entre o original e sua versão ${ }^{19}$.

Neste artigo, embasado em trabalho anterior, descreve-se o modelo RE-AIM para o público brasileiro. Como tal, o objetivo do presente artigo é apresentar o processo e os resultados da tradução e adaptação cultural do modelo RE-AIM para a realidade brasileira. A intenção é demonstrar como as dimensões do RE-AIM podem ser aplicadas na avaliação de programas, práticas $\mathrm{e}$ políticas focadas na promoção de estilos de vida saudáveis. Espera-se que esse modelo, traduzido e adaptado, possa contribuir para o planejamento, a avaliação e o monitoramento de intervenções, programas e políticas, assim como para nortear a atenção à saúde da população e os investimentos públicos, tornando-os mais produtivos, focados e articulados, gerando sinergia, ampliando resultados e o acesso à rede de serviços de maneira sustentável.

\section{MÉTODO}

O modelo RE-AIM foi traduzido e adaptado culturalmente seguindo $o$ protocolo proposto por Guillemin e colaboradores ${ }^{18}$. 0 processo foi subdividido em etapas conforme apresentado no fluxograma da Figura 1.

Figura 1: Resumo da metodologia empregada

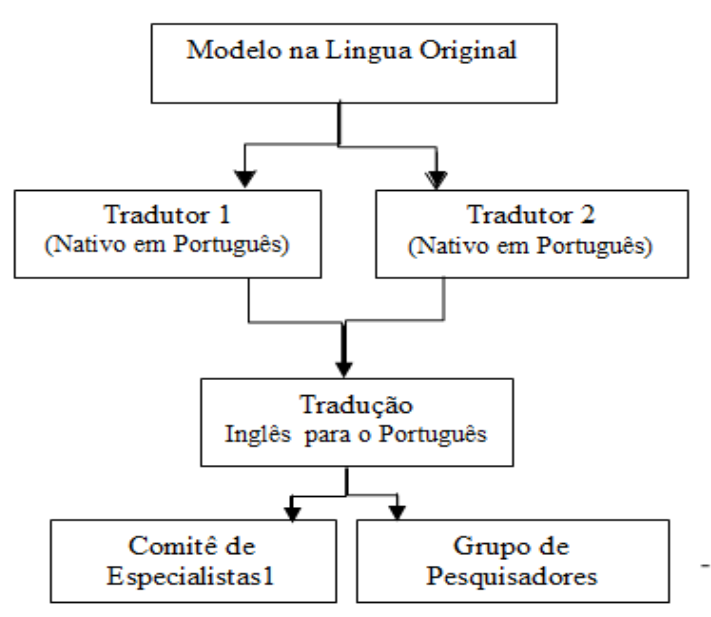


Pesquisadores de diferentes áreas temáticas vêm sugerindo que a ênfase não é dada só ao processo de tradução e retradução de instrumentos ${ }^{18,19,21}$. Sugerem que o processo seja uma combinação entre a avaliação semântica e a adequação ao contexto cultural e ao estilo de vida da população-alvo da versão ${ }^{18,21}$.
A Tabela 1 apresenta os aspectos avaliados e a estratégia para a avaliação adaptada e utilizada no processo segundo os conceitos dos autores. A equivalência da mensuração foi omitida, pois não era pertinente por se tratar de um modelo e não de um instrumento de mensuração.

Tabela 1. Principais etapas para avaliação da equivalência transcultural.

\begin{tabular}{ll}
\hline Aspecto avaliado & Estratégia para avaliação \\
\hline Equivalência conceitual & - Revisão bibliográfica envolvendo publicações da cultura do instrumento \\
& original e da população-alvo \\
& - Discussão com especialistas (Comitê 1) \\
& - Discussão com população-alvo (Grupo de pesquisadores) \\
Equivalência de itens & - Discussão com especialistas (Comitês 1 e 2) \\
Equivalência semântica & - Traduções \\
& - Retraduções \\
& - Avaliação da equivalência semântica entre as retraduções e o original \\
& - Discussão com a população-alvo (Grupo de pesquisadores) \\
& - Avaliação pelo grupo de pesquisa quanto à pertinência e adequação: \\
Equivalência operacional & - do veículo e formato das instruções; \\
& - do cenário de administração; \\
& - do modo de aplicação; e \\
Equivalência funcional & - do modo de categorização. \\
\hline
\end{tabular}

Fonte: Adaptado de Reichenheim e Moraes ${ }^{19}$.

A Equivalência conceitual e de itens envolveu uma discussão com especialistas (Comitês) com finalidade de explorar se os diferentes domínios abarcados pelo instrumento original na definição dos conceitos de interesse eram relevantes e pertinentes ao contexto brasileiro à luz da revisão bibliográfica acerca do RE-AIM. Também membros seletos e indivíduos representativos da população-alvo foram envolvidos na forma de um grupo de pesquisadores mono e bilíngues sendo apresentados a estes os conceitos traduzidos do RE-AIM feedback.

A equivalência semântica capacidade de transferência de sentido dos conceitos contidos no instrumento original para a versão - incluiu a tradução e a retradução do modelo obtidas pelos tradutores de forma independente e cega. Tendo em vista que a retradução é um passo importante e altamente recomendado por especialistas em pesquisa transcultural ${ }^{19,22}$, adicionalmente é também necessário que as versões do modelo sejam comparadas sistematicamente com as versões originais e retraduzidas ${ }^{19}$. Dessa forma, foi avaliada a equivalência entre as retraduções e o modelo original sob a perspectiva do significado referencial (denotativo) dos termos e palavras constituintes e o significado geral (conotativo) de cada item, transcendendo a literalidade das palavras nos usuários pretendidos do modelo acerca da aceitabilidade, compreensão e impacto emocional ${ }^{19}$.

O próximo passo envolveu o mesmo grupo de especialistas que participou da equivalência conceitual para identificar e encaminhar os problemas de cada uma das atividades anteriores, complementadas pelos tradutores e equipe de pesquisa encarregados da comparação das retraduções com o original. Foram então solucionados os problemas, realizadas modificações consensuais para melhor atender os critérios e criada a versãosíntese.

A versão foi então apresentada a um grupo de pesquisadores multiprofissionais 
para que fossem observados potenciais problemas de tradução e conceituais, além da clareza e adequação aos objetivos. Foram conduzidas avaliações interinas pela equipe de pesquisa em conjunto com especialistas convidados para tal. Com base nas evidências encontradas nesse pré-teste, foi realizada a equivalência operacional referente aos aspectos de utilização do modelo pela população-alvo. Posteriormente, realizaram-se ajustes semânticos finais na versão-síntese que foram apresentados nos resultados $e$ discutidos a seguir.

\section{RESULTADOS}

Como resultado da tradução e adaptação do modelo RE-AIM, apresentam-se a seguir as definições conceituais de cada uma das suas cinco dimensões: Reach (Alcance), Eficácia, Adoção, Implementação e Manutenção (Quadro 1).

Quadro 1 - Modelo RE-AIM: Dimensões traduzidas e adaptadas para o Brasil.

\begin{tabular}{|c|l|c|c|}
\hline $\begin{array}{c}\text { Dimensões do } \\
\text { RE-AIM }\end{array}$ & \multicolumn{1}{|c|}{ Definições } & $\begin{array}{c}\text { Nível de } \\
\text { Avaliação }\end{array}$ \\
\hline Alcance & $\begin{array}{l}\text { É o número absoluto, a proporção e a representatividade dos } \\
\text { indivíduos que estão dispostos a participar de uma determinada } \\
\text { iniciativa comparada àqueles que desistem ou àqueles potencialmente } \\
\text { elegíveis. }\end{array}$ & Individual \\
\hline $\begin{array}{c}\text { Efetividade ou } \\
\text { Eficácia }\end{array}$ & $\begin{array}{l}\text { É o impacto de uma intervenção sobre desfechos importantes } \\
\text { incluindo qualidade de vida, potenciais efeitos negativos e os } \\
\text { resultados econômicos. }\end{array}$ & Individual \\
\hline Adoção & $\begin{array}{l}\text { É o número absoluto, a proporção e a representatividade das } \\
\text { organizações e dos agentes de intervenção que estão dispostos a } \\
\text { iniciar um programa. }\end{array}$ & Organizacional \\
\hline Implementação & $\begin{array}{l}\text { No nível organizacional, refere-se à fidelidade dos agentes de } \\
\text { intervenção aos vários elementos de um protocolo de intervenção. } \\
\text { Isso inclui a consistência no fornecimento, conforme previsto, o tempo } \\
\text { e o custo da intervenção. No nível individual, refere-se à medida que } \\
\text { os participantes utilizam as estratégias da intervenção. }\end{array}$ & $\begin{array}{c}\text { Individual e } \\
\text { Monacional }\end{array}$ \\
\hline Manutenção & $\begin{array}{l}\text { No nível organizacional, é à medida que um programa ou política } \\
\text { torna-se institucionalizado ou parte das práticas de rotina e políticas } \\
\text { organizacionais. No nível individual, a manutenção tem sido definida } \\
\text { como os efeitos benéficos em longo prazo (seis ou mais meses após o } \\
\text { término da intervenção). }\end{array}$ & $\begin{array}{c}\text { Individual e } \\
\text { organizacional }\end{array}$ \\
\hline
\end{tabular}

\section{DISCUSSÃO}

Apresenta-se a seguir os conceitos-chave do RE-AIM, e as possíveis contribuições para o potencial impacto na saúde coletiva, no que se refere às intervenções.

\section{Alcance (Reach)}

Alcance (Reach) é definido como o número de participantes, a proporção da populaçãoalvo que participa e a representatividade dos participantes com relação à população-alvo. A proporção é operacionalizada como a taxa de participação, ou seja, o número de participantes divididos pelo número total de pessoas que são elegíveis para a participação. Dessa forma, o cálculo do Alcance (Reach) é demonstrado na Figura 2. A medida do alcance (Reach) é sempre realizada em nível individual.
Uma questão a se considerar em relação ao alcance é se a intervenção está realmente alcançando os grupos vulneráveis, com o objetivo de reduzir as disparidades na saúde com base na renda, idade, raça e etnia, Figura 2: Fórmula para o cálculo do Alcance (Reach).

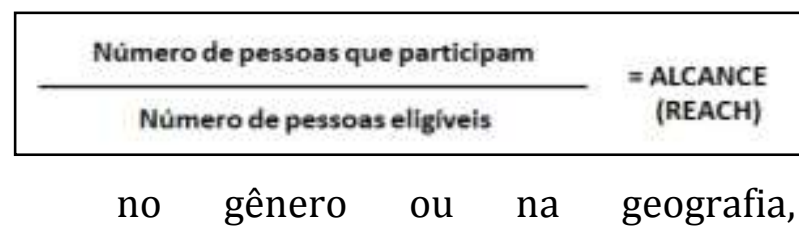
componentes essenciais para a equidade princípio fundamental do SUS. Para tal, se deve considerar que intervenções atraem diferentes grupos com base em vários fatores - necessidade ou benefício percebidos, custo para participação, relevância cultural, acesso à intervenção e outros. 


\section{Administração em Saúde Coletiva}

Assim, quando se deseja avaliar o potencial de alcance de uma intervenção ou o alcance de uma intervenção existente, as principais perguntas a serem realizadas incluem: (a) quantas pessoas são elegíveis para a participação (denominador)?; (b) quantas pessoas realmente participam e em que medida (numerador)?; (c) eles são representativos da população?; (d) são os indivíduos com maior risco aqueles que são alcançados?

A fim de avaliar a representatividade, é necessário monitorar as características das pessoas que participam. Com base nessas informações, podem-se conhecer as semelhanças e diferenças entre aqueles que participam, aqueles que são elegíveis e os que foram convidados, mas que não participam, assim como as características dos participantes em relação à população como um todo.

Se o alcance é baixo entre a população elegível, é preciso rever o processo e analisar quais poderiam ser as barreiras para a participação. Geralmente, descobrir-se-á que um ou mais fatores estão agindo como barreiras a esse fluxo. Fatores como o fato de que a população prioritária tem preocupação com relação a intervenção ou não valoriza o serviço ofertado; ou de que o "custo" da participação é muito alto, por exemplo, o tempo fora do trabalho para participar; ou ainda de que a intervenção não atende às necessidades da população pretendida.

A melhor maneira de descobrir o que está errado é se comunicar com as pessoas que se quer alcançar, ouvir o que elas têm a dizer e adaptar a intervenção ou os seus métodos de recrutamento para resolver essas preocupações.

\section{Efetividade/Eficácia}

Efetividade refere-se ao quanto a intervenção afeta uma mudança no desfecho primário de interesse, como ela afeta a qualidade de vida, se existem ou não quaisquer resultados imprevistos (positivos ou negativos), e como estes funcionam entre os subgrupos da população. Esses resultados pretendidos podem ser de médio ou longo prazo e geralmente são medidos em nível individual.

Particularmente na conceituação de efetividade do RE-AIM (ou eficácia altamente controlada em pesquisas clínicas randomizadas), a qualidade de vida é proposta como forma de se medir resultados que podem ser utilizados na comparação entre pesquisas focadas em diferentes comportamentos humanos. É também, sem dúvida, o resultado mais importante quando se considera uma intervenção de abordagem centrada no cliente/usuário/paciente ${ }^{12}$.

Um aspecto muitas vezes esquecido da efetividade inclui as consequências não intencionais - tanto os pontos positivos quanto negativos. Por exemplo, um programa de condicionamento físico para as mulheres pode aumentar a atividade física, mas também pode criar novas redes de apoio social que melhoram a qualidade de vida. 0 inverso pode também ser verdadeiro. Um programa de perda de peso pode melhorar a pressão arterial, mas as mudanças de estilo de vida que são necessárias podem causar conflito entre os membros da família.

A avaliação de um programa ou intervenção pode fornecer evidências diretas de que uma intervenção é efetiva. Após a seleção ou o desenvolvimento de uma intervenção, deve-se planejar antecipadamente a avaliação. Descrever um "modelo lógico" que detalhe os recursos disponíveis, as atividades, os produtos ou resultados e os resultados pretendidos pode ser uma ferramenta útil. Além disso, esse modelo lógico pode ajudar a ter como foco os resultados de curto, médio e longo prazo mais significativos e as principais medidas do processo, tais como as taxas de participação e a forma que a intervenção é realmente aplicada.

Além de se planejar antecipadamente a avaliação e de desenvolver um modelo lógico para apoiar o plano, quando se avalia a efetividade de um programa é vantajosa a identifição dos desfechos de interesse, considerando a possibilidade de múltiplos 


\section{Administração em Saúde Coletiva}

resultados, além de se ulilizar medidas válidas e confiáveis para a aferição de mudanças, medir a participação na intervenção e o controle da aplicação da intervenção.

\section{Adoção}

A adoção é definida em termos de nível organizacional ou de pessoal responsável por implementar e manter uma determinada intervenção. Assim, a adoção é o número de estruturas organizacionais ou de pessoal, a proporção da população-alvo dentre essas estruturas que participou e a representatividade das estruturas participantes.

Essencialmente, a adoção é a forma como as principais agências, organizações ou comunidades e seu pessoal estão dispostas e são capazes de aplicar a intervenção. Para tal, o desenvolvimento de parcerias com organizações que têm objetivos e interesses semelhantes à proposta pode aumentar significativamente a probabilidade de adoção de uma intervenção.

Outro aspecto da adoção que deve ser considerado é o planejar ou avaliar os esforços de promoção da saúde e a representatividade das organizações que participam ou adotam uma intervenção. Por exemplo, se o objetivo de um programa é aumentar a disponibilidade de espaços públicos para as crianças brincarem, e apenas as comunidades abastadas (em recursos) se aplicam para o recebimento dos subsídios para melhorar ou construir o espaço, isso significa que as comunidades mais vulneráveis socialmente não vão se beneficiar do programa. Sendo assim, o programa não alcança e nem promove acessibilidade ou equidade.

Quando se está avaliando a adoção real ou potencial de uma intervenção, as questões e os processos são semelhantes aos do Alcance (Reach), porém mais voltados para o nível organizacional e/ou o nível de pessoal. Quantas organizações são elegíveis para participação? A elegibilidade pode ser determinada por um conjunto de características que são importantes para a adoção. Ele pode ser restringido por localização, população atendida ou outros critérios. 0 número de organizações que são elegíveis e convidadas a participar é o denominador da adoção. Quantas organizações realmente participam? Esse é o seu numerador, conforme apresentado na Figura 3.

Figura 3: Fórmula para o cálculo da Adoção.

$$
\frac{\text { Número de organizações que participaram }}{\text { Número de organizações são eligiveis }}=\text { ADOÇão }
$$

Da mesma forma, em termos de pessoal, quais são os requisitos para a aplicação do programa ou intervenção? Quantos funcionários foram abordados e quantos participaram? Há diferenças entre as organizações e recursos humanos que adotam a intervenção e aqueles que não o fazem? As características das organizações e dos profissionais são importantes quando se considera a representação.

Acrescido a isso está o fato de que a adoção de uma intervenção por uma organização depende de como o novo programa se encaixa aos valores e à missão das organizações-alvo e, em muitos casos, o senso de urgência ou a vontade política para resolver o problema. Se a adoção da nova intervenção não é uma prioridade, é inconsistente com a missão ou irá resultar em uma interrupção significativa dos papéis e responsabilidades do pessoal, ela é improvável. Além disso, as organizações adotantes vão querer saber se a intervenção que se propõe funciona (efetividade/eficácia). Por outro lado, se a organização não tem recursos e profissionais experientes para ter sucesso ou se a intervenção é muito complexa e, assim o insucesso seja visível, é provável que a organização desista.

\section{Implementação}

A Implementação está relacionada ao grau em que uma intervenção é aplicada como pretendida, os custos associados a esta, incluindo o tempo e os custos da intervenção em nível organizacional. No nível individual, refere-se à forma como os participantes utilizam as estratégias da intervenção. A 


\section{Administração em Saúde Coletiva}

inclusão de custos ajuda no fornecimento de informações práticas às organizações e também permite análises de custoefetividade.

Cada intervenção tem elementos centrais ou componentes que são fundamentais para a sua eficácia. Compreender os elementos centrais de uma intervenção é fundamental para sua implementação. Elementos centrais são componentes da intervenção cruciais paraa eficácia da intervenção. Com base na teoria, na sequência lógica de atividades, nas principais estratégias e, em alguns casos, no nível de exposição, elementos centrais são as principais coisas que devem ser feitas para o sucesso da intervenção. Portanto, o primeiro passo para avaliar a implementação é identificar os elementos centrais. Isso é importante não só para o desenvolvimento de uma nova intervenção, mas também para se adotar ou adaptar uma intervenção existente, ou ainda para projetar a avaliação de uma intervenção.

Para a avaliação de um programa, devem-se conceber medidas de processo que captem dados sobre cada um dos elementos centrais e incluir medidas de garantia de qualidade. Para que se compreenda a efetividade de uma intervenção, é preciso compreender a extensão em que todos os recursos críticos ou os elementos fundamentais dessa intervenção foram implementados. Conceber uma avaliação que não descreve a implementação significa que não se sabe realmente $o$ que se está avaliando.

Ao se pensar sobre a implementação, é também importante considerar os custos desta, incluindo as competências necessárias dos profissionais ou capacitações, o custo de materiais, o custo para os participantes, como tempo e viagens, a compatibilidade com os recursos disponíveis, dentre outros aspectos.

Implementação é a chave para o sucesso. Muitas vezes, conversar com os atores que serão responsáveis pela implementação durante a fase de planejamento pode ajudar a antecipar os desafios que possam surgir. Envolve-los pode melhorar o compromisso destes e a aceitação da intervenção. Adaptar a intervenção e materiais para atender a cultura, a alfabetização e as normas sociais em sua comunidade sem retirar ou diluir os elementos centrais tambem é importante para facilitar a implementação de um programa.

\section{Manutenção}

A dimensão final do RE-AIM é a Manutenção. A manutenção pode ser considerada em termos organizacionais a sustentabilidade de um programa ao longo do tempo, ou, em âmbito individual, quando se refere à manutenção do resultado observado por participantes. Também é definida como o grau no qual a intervenção é mantida ou não após o financiamento formal, quando acabam os recursos provindos de uma pesquisa.

Questões importantes a serem consideradas acerca da manutenção são como incorporar o programa a uma organização para que ela seja sustentada ao longo do tempo e ajudar os participantes a ficarem engajados e sustentarem as mudanças positivas de comportamento ao longo do tempo.

Essas perguntas refletem os fatores que afetam a manutenção, uma vez que esta significa que um indivíduo ou uma organização realizou mudanças duradouras na forma como realiza certas atividades. Mudanças de longo prazo só são possíveis quando o benefício dessa mudança em relação ao custo de produção é benéfico tanto para os indivíduos que participaram quanto para as organizações que proporcionam a intervenção. Para tanto, é necessário avaliar como e quais fatores afetam a manutenção no nível organizacional e individual, e como se pode lidar com esses fatores.

Os fatores que afetam a manutenção no nível organizacional incluem recursos humanos, parcerias, financiamento e a própria organização em si. Os profissionais envolvidos é um fator importante para ajudar a garantir a manutenção de um 


\section{Administração em Saúde Coletiva}

programa. Sem pessoal adequado para executar o programa ou a intervenção, é praticamente impossível sustentar ou institucionalizar uma intervenção. Ter as atividades do programa incluídas como parte das descrições de funções e cargos pode ajudar o programa a ser promovido dentro da organização, difundido para todos os usuários em potencial, de forma a sobreviver à rotatividade de pessoal, aumentando a probabilidade de que seja mantido. Para tal, parcerias são recursos valiosos para ajudar uma organização na sustentabilidade de uma intervenção. Os parceiros podem ajudar a aumentar a conscientização da comunidade, a realizar formação continuada, a promover a sustentabilidade e a identificar novas fontes de financiamento, que são importantes para a manutenção de um programa.

No nível individual, a manutenção é o objetivo final de toda intervenção, sendo importante para ajudar a demonstrar a qualidade global de um programa. Recomendações para melhorar a manutenção em longo prazo no nível individual incluem: minimizar os recursos necessários, ensinar o automonitoramento, instituir políticas de incentivos e suporte, entrar em contato com os participantes periodicamente, referir os participantes para outros recursos da comunidade e incentivar grupos de apoio social. Além disso, é importante a realização de avaliações e acompanhamento para aprender com aqueles que foram bem-sucedidos e com aqueles que não foram, e adaptar as futuras intervenções a barreiras específicas para sua manutenção.

\section{Interação entre as dimensões do RE-AIM}

As cinco dimensões do RE-AIM trabalham juntas em sintonia sem que nenhuma seja considerada mais importante que a outra. A importância de cada dimensão pode mudar dependendo do objetivo predeterminado de cada programa.

O Alcance e a Adoção trabalham em conjunto, visando aumentar o número e a representatividade das populacões atendidas por programas de saúde.
Enquanto o Alcance está focado em aumentar a taxa de participacão e a representatividade dos participantes, a Adoção proporciona a possibilidade de aumentar exponencialmente o número de pessoas beneficiadas. Se um programa ou ação de governo tem um alto Alcance, mas uma baixa Adoção por entidades envolvidas, o programa é limitado na sua habilidade de atender a todos aqueles que poderiam ser beneficiados.

Dessa forma, programas ou ações que forem adotados por várias organizações, mas que não consigam atender aos mais necessitados, podem ter uma Adoção ampla com um pequeno Alcance dentro da população-alvo. Por isso é de suma importância um equilíbrio entre o Alcance e a Adoção, para se identificar barreiras potenciais e maximizar o potencial de Alcance e benefício para as populações mais necessitadas. Um número maior de organizações e mais profissionais aumentam o acesso, com o potencial de aumentar o Alcance de uma intervenção.

A Efetividade depende da Implementação. Uma das falhas comuns na avaliação de uma intervenção não é a avaliação dos resultados, mas $\operatorname{sim}$ a avaliação do processo de implementação. Muitas vezes, programas são adaptados de acordo com a realidade local, e se algumas dessas adaptações feitas para adequar os recursos, competências e conhecimentos disponíveis forem adaptações de elementos centrais do programa, isso pode levar a resultados decepcionantes. Portanto, um programa deve ser implementado com o máximo de fidelidade possível aos elementos centrais, para a verificação do funcionamento ou não.

Assim, enquanto o Alcance e a Adoção trabalham juntos para maximizar a capacidade dos programas de atenderem às populações mais carentes, a Implementação bem-sucedida de um programa pode levar a uma maior eficácia da intervenção, provendo assim o embasamento necessário para a Manunteção de um programa em longo prazo. 


\section{CONCLUSÃO}

A proposta deste artigo foi não somente traduzir um modelo, mas também adaptá-lo à realidade brasileira. Esse não é um processo simples, pois os valores refletidos por um instrumento e os significados das suas construções de componentes podem variar de uma cultura para outra. Portanto, procurou-se estabelecer a equivalência, cujo foco central, a adequação do modelo proposto com base em sua utilização em sua língua/cultura original para a sua utilização na língua/cultura brasileira, é uma perspectiva diferente da simples tradução de um instrumento.

O Modelo RE-AIM pode ser um valioso instrumento para planejamento e avaliação, uma vez que as dimensões do REAIM, assim como as reflexões sobre seus conceitos, se alinham com as doutrinas e os princípios do SUS: universalização, equidade, integralidade, descentralização e participação popular. Esses elementos se relacionam com as dimensões ao avaliar as peculiaridades e determinações locais, com ampla possibilidade de utilização pela gestão descentralizada político-administrativa, bem como pelo controle social.

O RE-AIM proporciona um método para avaliar o potencial ou o real impacto na saúde pública de programas e intervenções com grande potencial para colaborar na consolidação do SUS na prática, na medida em que considera seus princípios doutrinários.

O modelo RE-AIM pode ser aplicado em todos os níveis do Modelo Socioecológico, atentando para todos os níveis de influência que podem ser abordados, a fim de apoiar as escolhas de estilo de vida saudável em longo prazo, considerando que a formulação e a implementação de programas e intervenções em saúde devem ter como finalidade a melhoria das condições de vida e saúde dos diversos grupos da população, de modo a assegurar a universalização do acesso e a integralidade das ações de maneira resolutiva, articulada e dinâmica, tanto em âmbito individual quanto organizacional.

\section{REFERÊNCIAS}

1. Victora CG, Barreto ML, Leal M, Monteiro CA, Schmidt MI, Paim J, et al. Saúde no Brasil: condições de saúde e inovações nas políticas de saúde no Brasil: o caminho a percorrer. Lancet. 2011; 377(9782):90102.

2. Starfield B. Atenção primária: equilíbrio entre necessidades de saúde, serviços e tecnologia. Brasília: UNESCO, Ministério da Saúde; 2002.

3. Mendes JMR, Garcia MLT, Oliveira EFDA, Fernandes RMC. Gestão na saúde: da reforma sanitária às ameaças de desmonte do SUS. Textos Contextos (Porto Alegre). 2011; 10(2):331-44.

4. Ministério da Saúde (Br). Portaria GM no. 2488, de 21 de outubro de 2011. Aprova a Política Nacional de Atenção Básica, estabelecendo a revisão de diretrizes e normas para a organização da Atenção Básica, para a Estratégia Saúde da Família (ESF) e o Programa de Agentes Comunitários de Saúde (PACS). D.O.U. [Internet]. 2011 out 24 [acesso em 24 de jun 2013]. Disponível em: http://www.brasilsus.com.br/legislacoes/gm/11015 4-2488.html.

5. Pellegrini Filho A. Conferência mundial sobre determinantes sociais da saúde. Cad Saúde Pública. 2011; 27(11):2080-1.

6. Ministério da Saúde (Br). Secretaria de Vigilância em Saúde. Departamento de Análise de Situação de Saúde. Plano de ações estratégicas para o enfrentamento das doenças crônicas não transmissíveis (DCNT) no Brasil 2011-2022. Brasília (DF): Ministério da Saúde; 2011.

7. Ministério da Saúde (Br). Secretaria de Vigilância em Saúde, Política Nacional de Promoção da Saúde. Brasília (DF): Ministério da Saúde; 2006.

8. Malta DC, Morais Neto OLD, Silva Junior JBD. Apresentação do plano de ações estratégicas para o enfrentamento das doenças crônicas não transmissíveis no Brasil, 2011 a 2022. Epidemiol Serv Saúde. 2011; 20(4):425-38.

9. Fisher EB, Fitzgibbon ML, Glasgow RE, Haire-Joshu D, Hayman LL, Kaplan RM, et al. Behavior matters. Am J Prev Med. 2011; 40(5):15-30.

10. World Health Organization. Global status report on noncommunicable diseases 2010. Geneva: World Health Organization; 2011.

11. Glanz K, Bishop DB. The role of behavioral science theory in development and implementation of public health interventions. Ann Rev Public Health. 2010; 31:399-418.

12. Glasgow RE, Vogt TM, Boles SM. Evaluating the public health impact of health promotion interventions: the RE-AIM framework. Am J Public Health. 1999; 89(9):1322-7.

13. Green LW, Glasgow RE. Evaluating the relevance, generalization, and applicability of research issues in external validation and translation methodology. Eval Health Prof. 2006; 29(1):126-53.

14. Klesges LM, Estabrooks PA, Dzewaltowski DA, Bull SS, Glasgow RE. Beginning with the application in 
mind: designing and planning health behavior change interventions to enhance dissemination. Ann Behav Med. 2005; 29(2):66-75.

15. Glasgow RE, Tracy Orleans C, Wagner EH, Curry SJ, Solberg LI. Does the chronic care model serve also as a template for improving prevention? Milbank Q. 2001; 79(4):579-612.

16. Belza B, Toobert DJ, Glasgow RE. RE-AIM for program planning: overview and applications [Internet]. Washington: Center for Health Aging, National Concil on Aging; [2007] [acesso em 2013 out 25]. Disponível em: http://www.prchan.org/docs/RE-AIM_issue_brief.pdf.

17. Virginia Polytechnic Institute and State University. Reach effectiveness adoption implementation maintenance (RE-AIM) [Internet]. Virgínia: Human Nutrition, Foods and Exercise, College of Agriculture and Life Sciences; 2013 [acesso em 2013 out 25]. Disponível em: http://www.re-aim.org.

18. Guillemin F, Bombardier C, Beaton D. Crosscultural adaptation of health-related quality of life measures: literature review and proposed guidelines. J Clin Epidemiol. 1993; 46(12):1417-32.

19. Reichenheim ME, Moraes CL. Operacionalização de adaptação transcultural de instrumentos de aferição usados em epidemiologia. Rev. Saúde Pública. 2007; 41(4):665-73.

20. Grassi-Oliveira R, Stein LM, Pezzi JC. Tradução e validação de conteúdo da versão em português do Childhood Trauma Questionnaire. Rev. Saúde Pública. 2006; 40(2):249-55.

21. MattaI SR, Luiza VL, Azeredo TB. Adaptação brasileira de questionário para avaliar adesão terapêutica em hipertensão arterial. Rev. Saúde Pública. 2013; 47(2):292-300.

22. Brislin RW. Back-translation for cross-cultural research. J Cross Cult Psychol. 1970; 1(3):185-216.

\section{AGRADECIMENTOS}

Os autores agradecem ao Dr. Russell Glasgow pelo contínuo desenvolvimento e refinamento do modelo RE-AIM, pelo seu constante suporte como mentor, assim como pelas inestimáveis contribuições a este projeto.

\section{CONTRIBUIÇÕES}

Fabio Araujo Almeida contribuiu para o design do estudo, o plano de análise, a interpretação e a redação, e realizou a tradução (tradutor 2). Também foi responsável por apresentar os resultados da tradução e a adaptação para o Comitê de Especialistas e o grupo de pesquisadores.

Fabiana de Almeida Brito atuou no desenho do estudo, na interpretação, redação e foi responsável por analisar os resultados e realizar a tradução (tradutor 1). Também foi responsável por incorporar o feedback do Comitê de Especialistas e o grupo de pesquisadores para as versões do modelo.

Paul Andrew Estabrooks participou na interpretação e discussão dos resultados, e foi responsável por revisar a retradução como um dos colaboradores originais e contínuos do modelo REAIM.

Os três autores contribuíram para a versão final do modelo traduzido e adaptado. 